\title{
MicroRNA-29a functions as a potential tumor suppressor through directly targeting CDC42 in non-small cell lung cancer
}

\author{
YONGQIANG LI ${ }^{1}$, ZHI WANG ${ }^{2}$, YIJIANG LI $^{2}$ and RUIJUN JING ${ }^{2}$ \\ Departments of ${ }^{1}$ Emergency and ${ }^{2}$ Thoracic Surgery, The Second Affiliated Hospital \\ of Xi'an Medical University, Xi'an, Shaanxi 710038, P.R. China
}

Received August 31, 2015; Accepted January 17, 2017

DOI: $10.3892 / \mathrm{ol} .2017 .5888$

\begin{abstract}
The expression and function of microRNA-29a (miR-29a) have been investigated in various types of cancer. In the present study, the expression, function and underlying molecular mechanism of miR-29a were investigated in non-small cell lung cancer (NSCLC). The expression level of miR-29a in NSCLC was determined using reverse transcription-quantitative polymerase chain reaction (RT-qPCR). Cell proliferation, migration and invasion ability were determined using Cell Counting Kit-8, cell migration and invasion assays, respectively. Bioinformatics analysis and dual-luciferase reporter assays were performed to determine whether cell division cycle 42 (CDC42) is a direct target gene of miR-29a. To assess CDC42 mRNA and protein expression following transfection with miR-29a, RT-qPCR and western blotting were performed. Following knockdown of CDC42, functional assays were performed to investigate the roles of CDC42 in NSCLC. The results demonstrated that miR-29a was downregulated in NSCLC and the decreased expression level of miR-29a was significantly associated with advanced tumor-node-metastasis classification and metastasis. In addition, upregulation of miR-29a inhibited cell proliferation, migration and invasion in NSCLC, whereas downregulation of miR-29a had the opposite effects. Furthermore, CDC42 was identified as a direct target gene of miR-29a in vitro. miR-29a was demonstrated to function as a tumor suppressor in NSCLC by directly targeting CDC42 and may be investigated further as a target therapy for NSCLC.
\end{abstract}

\section{Introduction}

Human lung cancer, one of the most common types of aggressive malignancy, is the primary cause of cancer-associated

Correspondence to: Professor Ruijun Jing, Department of Thoracic Surgery, The Second Affiliated Hospital of Xi'an Medical University, 167 Fangdong Road, Xi'an, Shaanxi 710038, P.R. China E-mail: jingruijun_8687@126.com

Key words: microRNA-29a, non-small cell lung cancer, cell division cycle 42 , targeted therapy mortality worldwide $(1,2)$. In 2015 , it was estimated that there would be 221,200 new cases and 158,040 mortalities due to lung cancer in the USA (3). Previous studies have demonstrated that environmental deterioration, tobacco use, radon exposure and occupational carcinogens are risk factors for lung cancer (4-7). Lung cancer is divided into two major clinically relevant groups, small cell lung cancer and non-small cell lung cancer (NSCLC), according to histological analysis (8). NSCLC is the predominant type of lung cancer and accounts for $\sim 83 \%$ of all cases (9). NSCLC may be further divided into three histological subtypes: Squamous cell carcinoma, adenocarcinoma and large cell carcinoma (10). In spite of advances in the development of therapeutic strategies for patients with NSCLC, the prognosis of patients with NSCLC has remained poor over the past decade and the 5-year survival rate is $\leq 15 \%(11,12)$. It has been demonstrated that the primary causes of mortality in NSCLC are invasion and metastasis of cancerous cells (13). In addition, $>80 \%$ of patients with NSCLC are diagnosed at the advanced or distant stages (14). Therefore, it is essential to understand the underlying molecular mechanisms of NSCLC and develop therapeutic strategies for patients with NSCLC.

The dysregulation of microRNAs (miRNAs/miRs) has been demonstrated in NSCLC (15-17). miRNAs are a class of short (19-24 nucleotides in length) endogenous non-protein-coding single-stranded RNAs, which regulate $\sim 1 / 3$ of mRNAs in the human genome (18). miRNAs regulate mRNA expression by binding the 3' untranslated regions (3'UTRs) of target mRNAs and inducing either translational repression or transcript degradation (19). It has been demonstrated that miRNAs contribute to various physiological and pathological processes, including cell proliferation, differentiation, morphogenesis, cell cycle regulation, apoptosis, migration and invasion (20). miRNAs are classified as tumor-suppressive miRNAs and oncogenic miRNAs (21). Tumor suppressive miRNAs are downregulated in cancer and inhibit carcinogenesis and progression, whereas oncogenic miRNAs are upregulated in cancer, and enhance the initiation and development of cancer (22-24). Therefore, miRNAs have been extensively investigated in cancer research as therapeutic targets and biomarkers, as they regulate gene expression and cell biological activities.

In the present study, the expression level of miR-29a was assessed in NSCLC tissues and cell lines. In addition, whether the expression level of miR-29a was associated with the clinicopathological features of patients with NSCLC 
was investigated. Furthermore, the effect of miR-29a on cell proliferation, migration and invasion were determined, and the underlying molecular mechanism of its functions in NSCLC was investigated. The results of the present study may inform the development of a novel therapeutic strategy for NSCLC.

\section{Materials and methods}

Clinical specimens. The present study was approved by the Ethics Board of The Institute of The Second Affiliated Hospital of Xi'an Medical University (Xi'an, China). Written informed consent was also obtained from each patient involved in the present study. A total of 62 pairs of NSCLC tissue specimens and corresponding adjacent non-tumor lung tissues were obtained from patients who had undergone surgery at The Second Affiliated Hospital of Xi'an Medical University between January 2012 and November 2014. Tissues were immediately snap-frozen in liquid nitrogen and stored at $-80^{\circ} \mathrm{C}$. No patient had received radiotherapy or chemotherapy prior to surgery. The complete clinical data for patients with NSCLC are presented in Table I.

Cell culture. The human NSCLC cell lines H23, H522, H1299 and A549, and non-tumorigenic bronchial epithelium cell line BEAS-2B were purchased from the American Type Culture Collection (Manassas, VA, USA). The human embryonic kidney cell line HEK293T was purchased from the Cell Bank of the Type Culture Collection of the Chinese Academy of Sciences (Shanghai, China). The NSCLC cell lines and HEK293T cell line were maintained in RPMI-1640 medium (Gibco; Thermo Fisher Scientific, Inc., Waltham, MA, USA) supplemented with $10 \%$ fetal bovine serum (FBS; Gibco; Thermo Fisher Scientific, Inc.), $100 \mathrm{U} / \mathrm{ml}$ penicillin and $100 \mathrm{mg} / \mathrm{ml}$ streptomycin (Gibco; Thermo Fisher Scientific, Inc.) in a cell incubator with $5 \% \mathrm{CO}_{2}$ at $37^{\circ} \mathrm{C}$. BEAS-2B cells were maintained in LHC-9 medium (Gibco; Thermo Fisher Scientific, Inc.) containing $10 \% \mathrm{FBS}$ at $37^{\circ} \mathrm{C}$.

Cell transfection. miR-29a mimics, negative control (NC), miR-29a inhibitor, NC inhibitor, cell division cycle 42 (CDC42) small interfering RNA (siRNA) and NC siRNA were obtained from Shanghai GenePharma Co., Ltd. (Shanghai, China). Cells were seeded into 6-well plates and cultured until the cell density reached $70-90 \%$. Cell transfection was performed using Lipofectamine ${ }^{\mathrm{TM}} 2000$ (Invitrogen; Thermo Fisher Scientific, Inc.), according to the manufacturer's protocol. Cell transfection efficiency was determined using reverse transcription-quantitative polymerase chain reaction (RT-qPCR).

$R N A$ isolation and $R T-q P C R$. Total RNA from tissues and cells was isolated using TRIzol ${ }^{\circledR}$ reagent (Invitrogen; Thermo Fisher Scientific, Inc.), according to the manufacturer's protocol. The miR-29a expression level was determined using an SYBR PrimeScript miRNA RT-PCR kit (Takara Bio, Inc., Otsu, Japan) with U6 as the internal control. The thermocycling conditions were as follows: $42^{\circ} \mathrm{C}$ for $5 \mathrm{~min} ; 95^{\circ} \mathrm{C}$ for $10 \mathrm{sec}$; and 40 cycles of $95^{\circ} \mathrm{C}$ for $5 \mathrm{sec}, 55^{\circ} \mathrm{C}$ for $30 \mathrm{sec}$ and $70^{\circ} \mathrm{C}$ for $30 \mathrm{sec}$. The expression of CDC42 mRNA was determined using a standard SYBR PrimeScript miRNA RT-PCR kit (Takara Bio, Inc.) with GAPDH as the control. The thermocycling conditions were as follows: $42^{\circ} \mathrm{C}$ for $5 \mathrm{~min} ; 95^{\circ} \mathrm{C}$ for $10 \mathrm{sec}$; and 40 cycles of $95^{\circ} \mathrm{C}$ for $5 \mathrm{sec}, 55^{\circ} \mathrm{C}$ for $30 \mathrm{sec}$ and $70^{\circ} \mathrm{C}$ for $30 \mathrm{sec}$. RT-qPCR was performed using an ABI 7500 Real-time PCR detection system (Applied Biosystems; Thermo Fisher Scientific, Inc.). The primer sequences were as follows: miR-29a forward, 5'-ACACTCCAGCTGGGA CTGATTTCTTTTGGT-3' and reverse, 5'-CTCAACTGG TGTCGTGGAGTCGGCAATTCAGTTGAGTCAGGTGT-3'; U6 forward, 5'-CTCGCTTCGGCAGCACA-3' and reverse, 5'-AACGCTTCACGAATTTGCGT-3'; CDC42 forward, 5'-ACGACCGCTGAGTTATCCAC-3' and reverse, 5'-TAT GGGCCTTGTCTCACACG-3'; and GAPDH forward, 5'-TGC ACCACCAACTGCTTAGC-3' and reverse, 5'-GGCATGCAC TGTGGTCATGAG-3'. RT-qPCR results were calculated using the $2^{-\Delta \Delta C q}$ method (25). Each sample was analyzed in triplicate and repeated $>3$ times.

Cell Counting kit-8 (CCK-8) assay. In vitro cell proliferation was monitored using a CCK-8 assay (Dojindo Molecular Technologies, Inc., Kumamoto, Japan), according to the manufacturer's protocol. Transfected cells were collected and seeded into 96-well plates at a density of 3,000 cells/well. Quantification of cell proliferation was performed daily for 4 days. A $10 \mu \mathrm{l}$ volume of CCK-8 assay solution was added to each well prior to incubation at $37^{\circ} \mathrm{C}$ for $2 \mathrm{~h}$ in a cell incubator. The absorbance at $450 \mathrm{~nm}$ of each well was determined using a spectrophotometer. Each sample was analyzed in triplicate.

Cell migration and invasion assays. In vitro cell migration and invasion assays were performed using Transwell chambers (EMD Millipore, Billerica, MA, USA) with an $8-\mu \mathrm{m}$ pore polycarbonate membrane. For the cell migration assay, $5 \times 10^{4}$ transfected cells in $100 \mu \mathrm{l}$ FBS-free RPMI-1640 medium were plated in the upper chamber. A $500 \mu 1$ volume of RPMI-1640 medium containing $20 \%$ FBS was added to the lower chamber as a chemoattractant. For the cell invasion assay, Transwell chambers were pre-coated with Matrigel (BD Biosciences, San Jose, CA, USA). Otherwise, the cell invasion assays were performed according to the procedure of the cell migration assay. After $24 \mathrm{~h}$ of incubation, cells were fixed with $95 \%$ ethanol and stained with $0.1 \%$ crystal violet (Beyotime Institute of Biotechnology, Haimen, China) for $20 \mathrm{~min}$. Non-migrating and non-invading cells were carefully scraped from upper chambers using cotton wool. Cells were counted using a light microscope. Each sample was repeated $\geq 3$ times.

Western blotting. Cells were lysed with radioimmunoprecipitation buffer (Thermo Fisher Scientific, Inc.). A bicinchoninic acid assay (Thermo Fisher Scientific, Inc.) was used to determine protein concentrations. Equal amounts of proteins $(20 \mu \mathrm{g})$ were separated by SDS-PAGE (10\% gels; Beyotime Institute of Biotechnology) and transferred onto polyvinylidene fluoride (EMD Millipore) membranes. The membranes were blocked with 5\% non-fat dry milk and incubated with mouse anti-human CDC42 monoclonal primary antibody (dilution, 1:500; cat. no. sc-8401; Santa Cruz Biotechnology, Dallas, TX, USA) and rabbit anti-human GAPDH monoclonal primary antibody (dilution, 1:1,000; cat no. 2118; Cell Signaling Technology, Inc., Danvers, MA, USA). Following incubation 
at $4^{\circ} \mathrm{C}$ overnight, the membranes were incubated with corresponding horseradish peroxidase-conjugated secondary antibody (both dilution, 1:5,000; CDC42 cat. no. sc-2005; GAPDH cat. no. sc-2054; both Santa Cruz Biotechnology, Dallas, TX, USA) for $1 \mathrm{~h}$ at room temperature. The membranes were visualized with enhanced chemiluminescence solution (Pierce; Thermo Fisher Scientific, Inc.) and analyzed using a FluorChem imaging system (version 4.1.0; Alpha Innotec, San Leandro, CA, USA). Each sample was repeated $\geq 3$ times.

miRNA target prediction. The potential target genes of miR-29a were predicted using TargetScan (www.Targetscan .org) and miRanda (www.microrna.org/microrna/home.do).

Dual-luciferase reporter assay. The luciferase reporter plasmids PGL3-CDC42-3'UTR wild-type (Wt) and PGL3-CDC42-3'UTR mutant (Mut) were purchased from Shanghai GenePharma Co., Ltd. HEK293T cells were seeded into 12-well plates and cultured as described above until the cell density reached $90 \%$. Cells were transfected with miR-29a mimics or NC, and co-transfection with PGL3-CDC42-3'UTR Wt or PGL3-CDC42-3'UTR Mut using Lipofectamine 2000 was performed according to the manufacturer's protocol. After transfection for $48 \mathrm{~h}$, firefly luciferase activity and Renilla luciferase activity were detected using the Dual-Luciferase Reporter Assay system (Promega Corporation, Madison, WI, USA), according to the manufacturer's protocol. The firefly luciferase activity was normalized to the Renilla luciferase activity. Each assay was replicated three times.

Statistical analysis. Data are presented as the mean \pm standard deviation, and were analyzed using the Student's $t$ test or one-way analysis of variance using SPSS software (version 17; SPSS Inc., Chicago, IL, USA). P $<0.05$ was considered to indicate a statistically significant difference.

\section{Results}

Expression of miR-29a in NSCLC tissues and cell lines. To investigate the potential function of miR-29a in NSCLC, the miR-29a expression level in NSCLC tissue specimens and corresponding adjacent non-tumor lung tissues was determined. The expression level of miR-29a was significantly decreased in NSCLC tissue compared with adjacent non-tumor lung tissue ( $\mathrm{P}<0.05$; Fig. 1A). The expression of miR-29a was also measured in NSCLC cell lines and the non-tumorigenic bronchial epithelium cell line BEAS-2B. Analysis using RT-qPCR revealed that the expression of miR-29a was downregulated in all four NSCLC cell lines compared with BEAS-2B cells $(\mathrm{P}<0.05$; Fig. 1B). These results suggested that miR-29a contributes to the initiation and progression of NSCLC.

miR-29a expression and clinicopathological features in NSCLC. To investigate the clinical significance of miR-29a expression in NSCLC, statistical analysis was used to explore the association between miR-29a and clinicopathological factors. As presented in Table I, it was identified that the low expression level of miR-29a was significantly associated with advanced tumor-node-metastasis (TNM) classification $(\mathrm{P}=0.003)$ and metastasis $(\mathrm{P}=0.020)$. However, no significant
Table I. Comparison of miR-29a expression in NSCLC and clinicopathological features.

\begin{tabular}{|c|c|c|c|c|}
\hline \multirow[b]{2}{*}{ Clinical feature } & \multirow[b]{2}{*}{$\mathrm{n}$} & \multicolumn{2}{|c|}{$\begin{array}{c}\text { miR-29a } \\
\text { expression }\end{array}$} & \multirow[b]{2}{*}{ P-value } \\
\hline & & Low & High & \\
\hline Gender & & & & 0.191 \\
\hline Male & 30 & 21 & 9 & \\
\hline Female & 42 & 23 & 19 & \\
\hline Age, years & & & & 0.449 \\
\hline$<60$ & 32 & 18 & 14 & \\
\hline$>60$ & 40 & 26 & 14 & \\
\hline Smoking history, years & & & & 0.117 \\
\hline$<10$ & 25 & 15 & 10 & \\
\hline$>10$ & 47 & 29 & 18 & \\
\hline TNM classification & & & & 0.003 \\
\hline I-II & 38 & 17 & 21 & \\
\hline III-IV & 34 & 27 & 7 & \\
\hline Tumor differentiation & & & & 0.212 \\
\hline $\mathrm{I}+\mathrm{II}$ & 45 & 25 & 20 & \\
\hline III+IV & 27 & 19 & 8 & \\
\hline Metastasis & & & & 0.020 \\
\hline No & 29 & 13 & 16 & \\
\hline Yes & 43 & 31 & 12 & \\
\hline
\end{tabular}

miR-29a, microRNA-29a; NSCLC, non-small cell lung cancer; TNM, tumor-node-metastasis.

association between miR-29a expression level and other clinicopathological features (gender, age, smoking history and tumor differentiation) was identified $(\mathrm{P}>0.05)$.

miR-29a inhibits NSCLC cell proliferation. To investigate the roles of miR-29a expression in NSCLC, miR-29a mimics, NC siRNA, miR-29a inhibitor and NC inhibitor were transfected into NSCLC cells. Among the four NSCLC cell lines, the expression level of miR-29a in H522 cells was the lowest and that in A549 cells was the highest. Consequently, H522 was transfected with miR-29a mimics or NC, and A529 was transfected with miR-29a inhibitor or NC inhibitor. Following transfection for $48 \mathrm{~h}$, the cell transfection efficiency was assessed using RT-qPCR. As presented in Fig. 2A, miR-29a was significantly upregulated in H522 cells transfected with miR-29a mimics, whereas miR-29a was downregulated in A549 cells transfected with miR-29a inhibitor $(\mathrm{P}<0.05)$.

The effect of miR-29a on cell proliferation was measured using a CCK-8 assay. The cell proliferation assay demonstrated that miR-29a mimics inhibited H522 cell proliferation and miR-29a inhibitor enhanced A549 cell proliferation $(\mathrm{P}<0.05$; Fig. 2B). These results indicate that miR-29a inhibits cell proliferation in vitro.

miR-29a inhibits NSCLC cell migration and invasion. In order to determine whether miR-29a was able to regulate NSCLC 
A

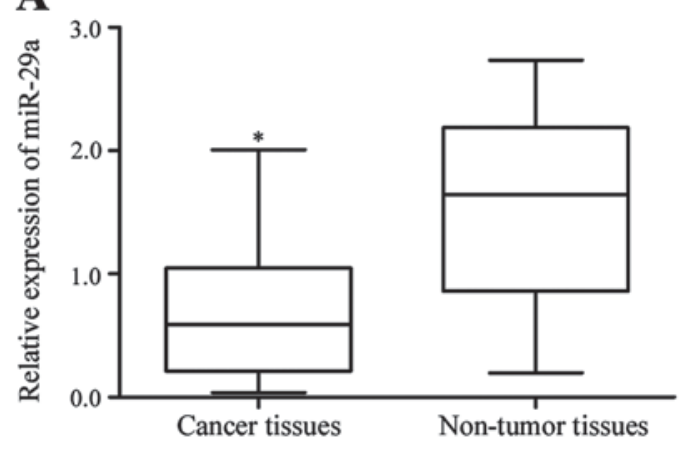

B

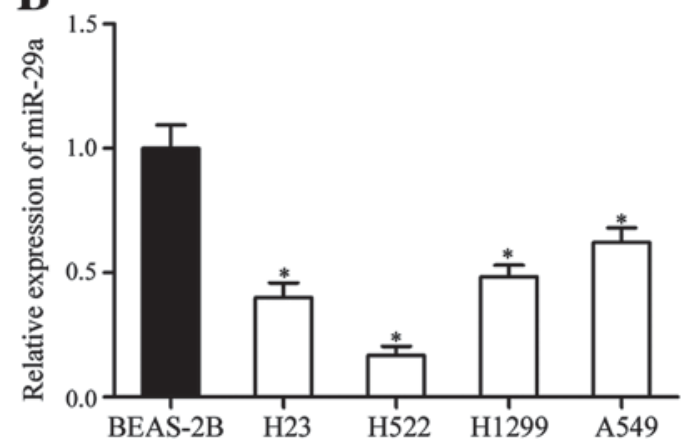

Figure 1. miR-29a is downregulated in NSCLC. (A) miR-29a was significantly downregulated in NSCLC tissues compared with corresponding adjacent non-tumor lung tissues. ${ }^{*} \mathrm{P}<0.05$ vs. non-tumor tissue. (B) Expression of miR-29a was significantly decreased in all four NSCLC cell lines compared with the non-tumorigenic bronchial epithelium cell line BEAS-2B. * $\mathrm{P}<0.05$ vs. BEAS-2B cells. miR-29a, microRNA-29a; NSCLC, non-small cell lung cancer.

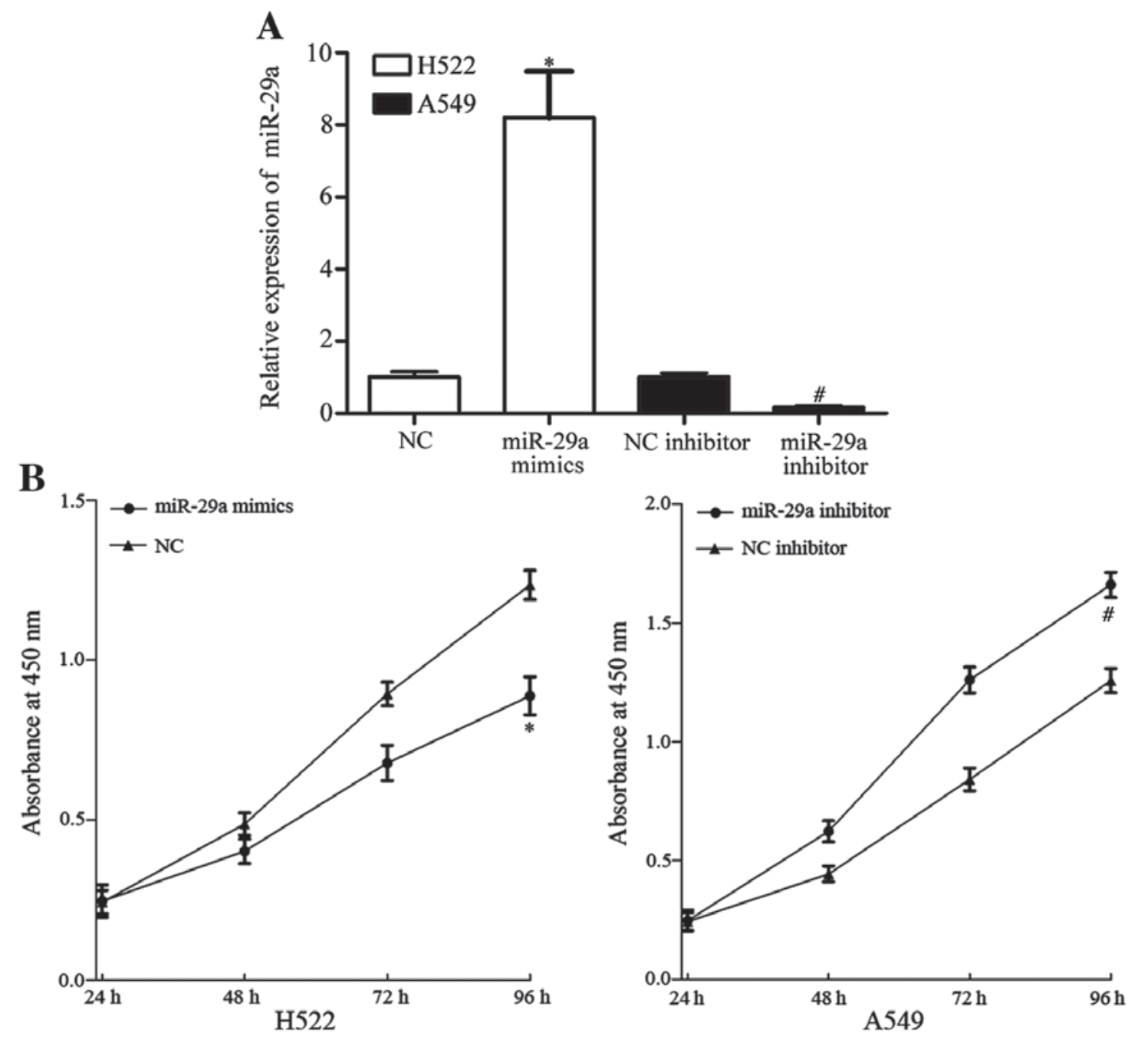

Figure 2. miR-29a inhibits NSCLC cell proliferation. (A) miR-29a was significantly upregulated in H522 cells after transfection with miR-29a mimics, whereas miR-29a was significantly downregulated in A549 cells after transfection with miR-29a inhibitor. ${ }^{*} \mathrm{P}<0.05$ vs. NC; ${ }^{\prime} \mathrm{P}<0.05$ vs. NC inhibitor. (B) A Cell Counting Kit- 8 assay revealed that miR-29a mimics inhibited $\mathrm{H} 522$ cell proliferation and that miR-29a inhibitor promoted A549 cell proliferation. ${ }^{*} \mathrm{P}<0.05$ vs. NC; "P<0.05 vs. NC inhibitor. miR-29a, microRNA-29a; NSCLC, non-small cell lung cancer; NC, negative control.

cell migration and invasion, cell migration and invasion assays were performed using Transwell chambers.

miR-29a mimics suppressed the cell migratory and invasive ability of H522 cells $(\mathrm{P}<0.05$; Fig. 3A). Furthermore,
miR-29a inhibitor increased the cell migratory and invasive ability of A549 cells ( $\mathrm{P}<0.05$; Fig. 3B). These results indicate that miR-29a was able to inhibit NSCLC cell migration and invasion ability in vitro. 
A

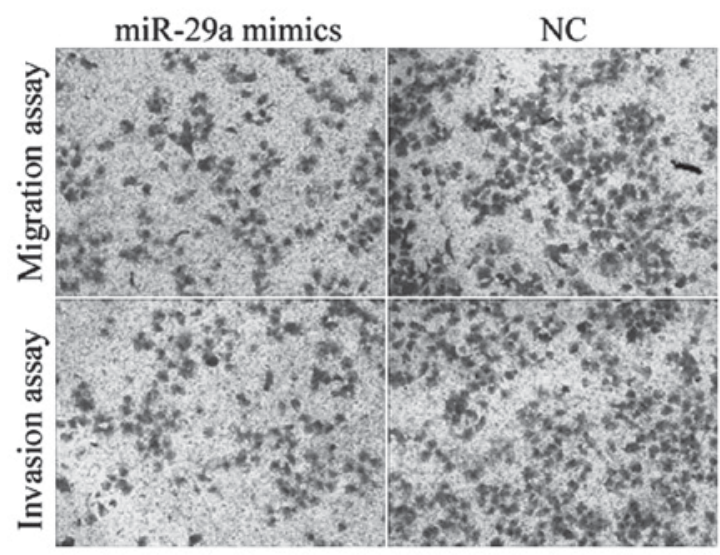

B

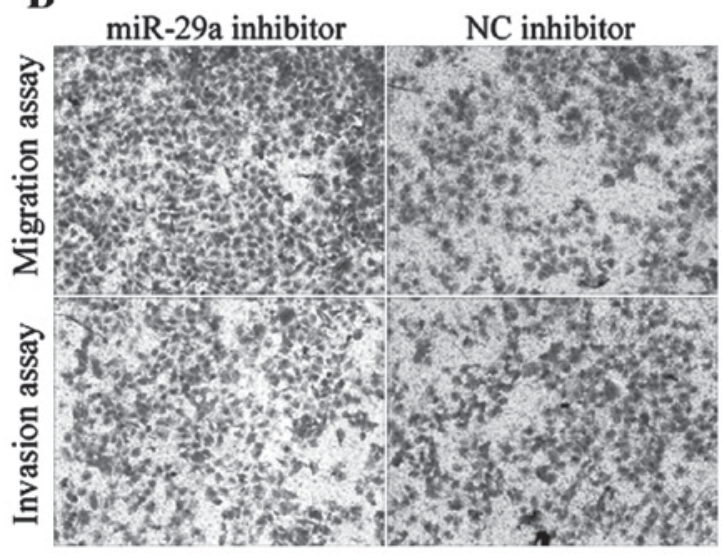

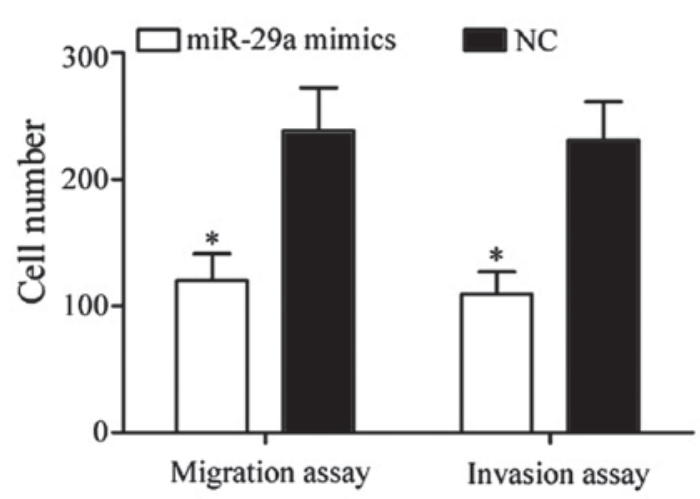

A549

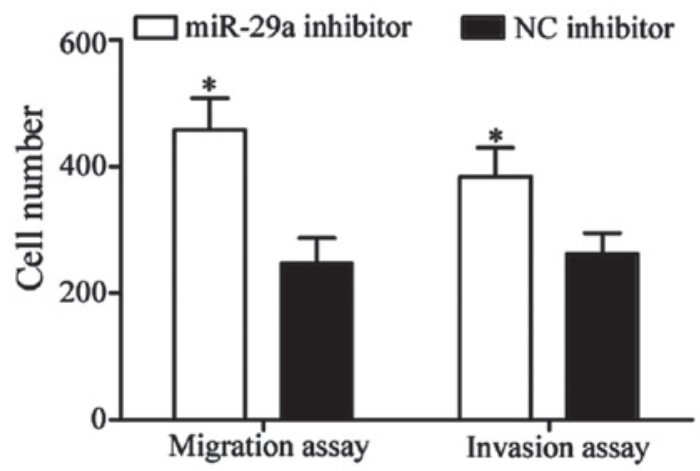

Figure 3. miR-29a inhibits NSCLC cell migration and invasion. (A) In H522 cells, miR-29a mimics inhibited cell migration and invasion compared with cells transfected with NC. * $\mathrm{P}<0.05$ vs. NC. (B) In A549 cells, miR-29a inhibitor enhanced cell migration and invasion compared with cells transfected with NC inhibitor. "P<0.05 vs. NC inhibitor. miR-29a, microRNA-29a; NSCLC, non-small cell lung cancer; NC, negative control.

CDC42 is a direct target gene of miR-29a in vitro. To investigate the molecular mechanism of miR-29a on NSCLC carcinogenesis, bioinformatics analysis was performed. TargetScan (www.targetscan.org) and miRanda (www .microrna.org/microrna) predicted that CDC42 was a direct target gene of miR-29a (Fig. 4A).

To investigate whether CDC42 was a direct target of miR-29a, a dual-luciferase reporter assay was used. The results demonstrated that miR-29a led to a significant decrease in pGL3-CDC42-3'UTR site $1 \mathrm{Wt}$ and pGL3-CDC42-3'UTR site $2 \mathrm{Wt}$ luciferase activity in HEK293T cells $(\mathrm{P}<0.05$; Fig. 4B). Mutation of miR-29a-binding sites (site 1 Mut or site 2 Mut) restored wild-type luciferase activity of CDC42-3'UTR in HEK293T cells. These results indicate that CDC42 is a direct target gene of miR-29a.

miR-29a regulates CDC42 expression at the post-transcriptional level. To explore the regulatory effect of miR-29a on CDC42, RT-qPCR and western blotting were performed to detect the expression of CDC42 at the mRNA and protein level in response to the alterations in miR-29a levels. CDC42 mRNA levels were not significantly altered during these treatments (P>0.05; Fig. 5A). However, western blot analysis revealed that CDC42 protein expression was significantly downregulated in H522 cells transfected with miR-29a mimics, whereas
A $\begin{array}{lcc}\text { Position 578-584 of CDC42 3' UTR } & 5, & \text {...AACAUGUUCCCCAUCUGGUGCUC.. } \\ \text { hsa-miR-29a } & 3, & \text { AUUGGCUAAAGUCUACCACGAU. } \\ & \text { Site } 1 & \\ \text { Position 997-1004 of CDC42 3' UTR } & 5, & \text {...AGGAAAUACGAGGGGUGGUGCUA.. } \\ \text { hsa-miR-29a } & 3, & \text { AUUGGCUAAAGUCUACCACGAU. }\end{array}$

B

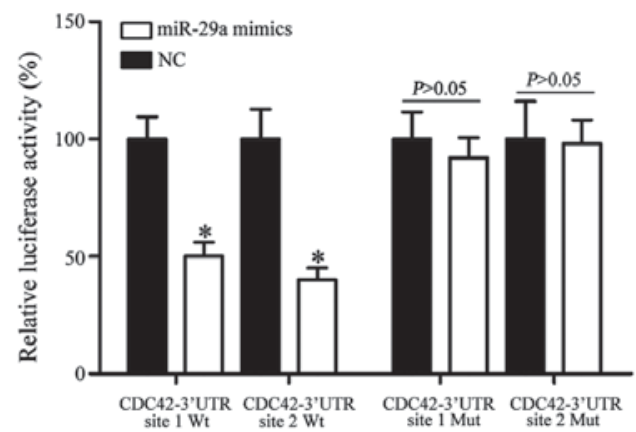

Figure 4. CDC42 is a direct target of miR-29a in vitro. (A) Two putative miR-29a-binding sites were identified at nucleotides 578-584 (site 1) and 997-1004 (site 2) of the CDC42 3'UTR. (B) A dual-luciferase reporter assay demonstrated that miR-29a significantly inhibited the PGL3-CDC42-3'UTR site $1 \mathrm{Wt}$ and PGL3-CDC42-3'UTR site $2 \mathrm{Wt}$ luciferase activity, but not the PGL3-CDC42-3'UTR site 1 Mut and PGL3-CDC42-3'UTR site 2 Mut luciferase activity in HEK293T cells. ${ }^{*} \mathrm{P}<0.05$ vs. NC. CDC42, cell division cycle 42; miR-29a, microRNA-29a; 3'UTR, 3' untranslated region; Wt, wild-type; Mut, mutant; NC, negative control; hsa, Homo sapiens. 


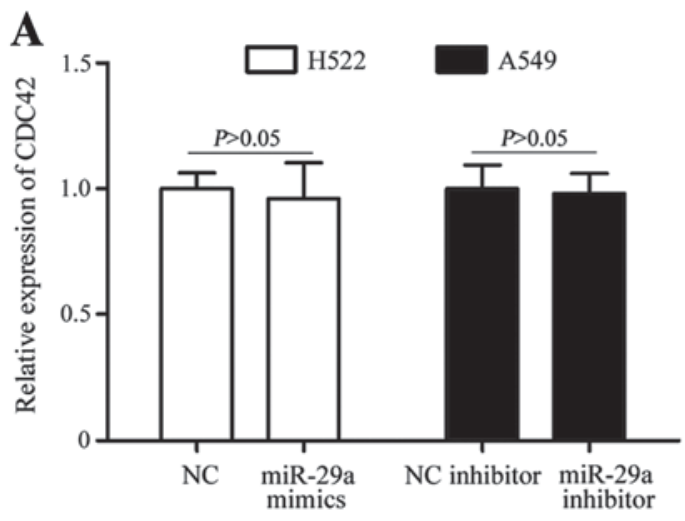

B
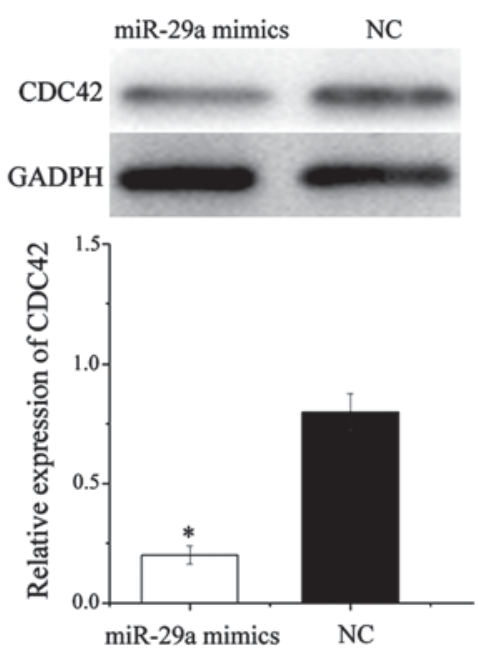

A549

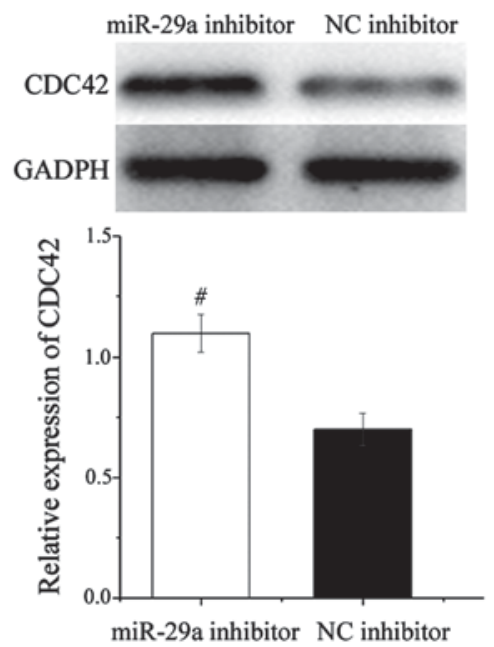

Figure 5. miR-29a regulates CDC42 expression at the post-transcriptional level. (A) In H522 cells, no differences were observed in CDC42 mRNA levels between cells transfected with miR-29a mimics and NC. In A549 cells, no differences were observed in CDC42 mRNA levels between cells transfected with miR-29a inhibitor and NC inhibitor. (B) Western blot analysis demonstrated that miR-29a mimics decreased CDC42 protein expression and miR-29a inhibitor increased CDC42 protein expression. GAPDH was used as a loading control. ${ }^{\text {P }}<0.05$ vs. NC; ${ }^{\text {P }<0.05 ~ v s . ~ N C ~ i n h i b i t o r ; ~ . ~ m i R-29 a, ~ m i c r o R N A-29 a ; ~ C D C 42, ~}$ cell division cycle 42; NC, negative control.

miR-29a inhibitor significantly increased CDC42 protein expression in A549 cells ( $\mathrm{P}<0.05$; Fig. 5B). These results indicate that miR-29a negatively regulates CDC42 expression at the post-transcriptional level.

CDC42 is involved in miR29a-induced effects in NSCLC cells. The endogenous expression of CDC42 was detected in H522, A549 and BEAS-2B cells. Western blot analysis demonstrated that the expression level of CDC42 was significantly upregulated in H522 and A549 cells compared with BEAS-2B cells $(\mathrm{P}<0.05$; Fig. 6A). These results are in accordance with the downregulation of miR-29a in NSCLC.

To explore further whether the functional effects of miR-29a on NSCLC cell lines was exerted via CDC42, functional assays were performed in $\mathrm{H} 522$ and A549 cells following transfection with CDC42 siRNA or NC siRNA. The transfection efficiency was measured using western blot analysis. The expression level of CDC42 was markedly decreased in H522 and A549 cells transfected with CDC42 siRNA compared with the NC siRNA groups (Fig. 6B).

The CCK-8 assay demonstrated that silencing of CDC42 decreased cell proliferation in $\mathrm{H} 522$ and A549 cells $(\mathrm{P}<0.05$; Fig. 6C). Furthermore, the cell migration and invasion assays revealed that knockdown of CDC42 in H522 and A549 cells significantly suppressed cell migration and invasion ability, in comparison with the NC siRNA-transfected cells $(\mathrm{P}<0.05$; Fig. 6D and E). These results indicate that the roles of $\mathrm{CDC} 42$ siRNA are similar to the functions exerted by miR-29a in NSCLC cells, indicating that CDC42 is a functional target of miR-29a in vitro.

\section{Discussion}

miR-29a is a member of the miR-29 family, which includes miR-29a, miR-29b-1, miR-29b-2 and miR-29c. These miRNAs are encoded in two clusters: miR-29b-1 and miR-29a in 7q32, and miR-29b-2 and miR-29c in 1q32 (26,27). miR-29a has been demonstrated to be downregulated in various types of cancer, including prostate cancer (28), gastric cancer (29), esophageal carcinoma (30), acute myeloid leukemia (31) and hepatocellular carcinoma (32). However, miR-29a was identified to be upregulated in colorectal cancer (33). These conflicting studies indicated that the expression of miR-29a in cancer has tissue-specificity. In the present study, it was demonstrated that the expression level of miR-29a was decreased in NSCLC tissues and cell lines. In addition, the decreased expression level of miR-29a was significantly associated with advanced TNM classification and metastasis. These results indicated that miR-29a may have a tumor-suppressive capacity in the carcinogenesis and progression of NSCLC.

Abnormal expression of miR-29a was demonstrated to be involved in the malignant phenotype of cancer. For example, in prostate cancer, upregulated miR-29a significantly inhibited cell proliferation and enhanced cell apoptosis by directly targeting histone lysine demethylase 5B (28). In renal cell carcinoma, miR-29a expression was decreased, and functioned as a cell migration and invasion suppressor by targeting lysine oxidase homolog 2 (34). Tréhoux et al (35) demonstrated that miR-29a suppressed cell proliferation, migration and invasion, and sensitized cells to gemcitabine by directly targeting mucin 1 in pancreatic cancer. In gastric cancer, enforced expression of miR-29a significantly reduced cell migration and invasion ability via blockade of roundabout guidance receptor 1 (29). In addition, miR-29 inhibited gastric cancer cell proliferation and would healing by downregulation of cyclin-dependent kinase (CDK) 2, CDK4 and CDK6 (36). In oral squamous cell carcinoma, miR-29a negatively regulated matrix metalloproteinase 2 (MMP2) expression to inhibit cell invasion and enhance apoptosis (37). However, in colorectal cancer, miR-29a promoted cell migration and invasion via targeting Krüppel-like factor 4 to regulate expression of MMP2 and epithelial cadherin (33). These conflicting studies suggest that 
A
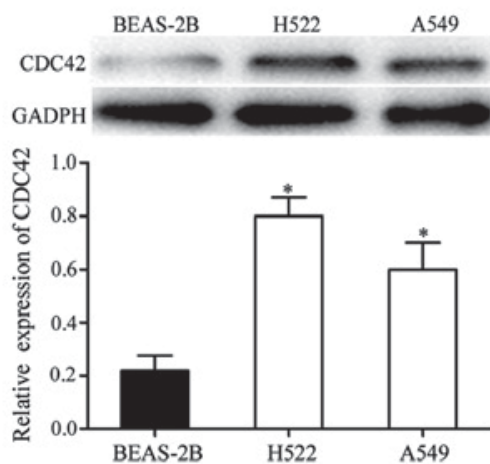

B

H522

A549

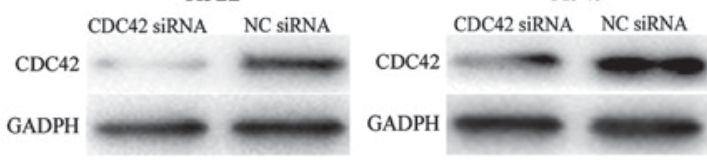

C
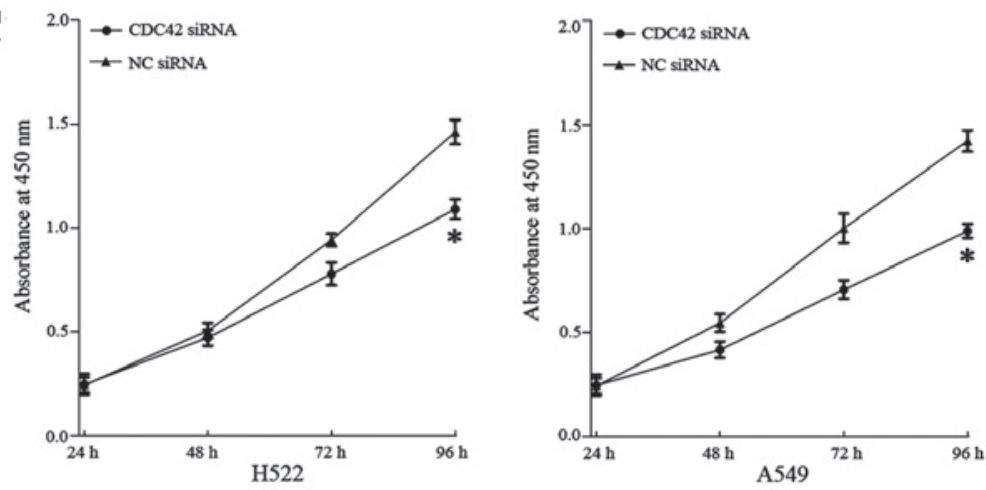

D

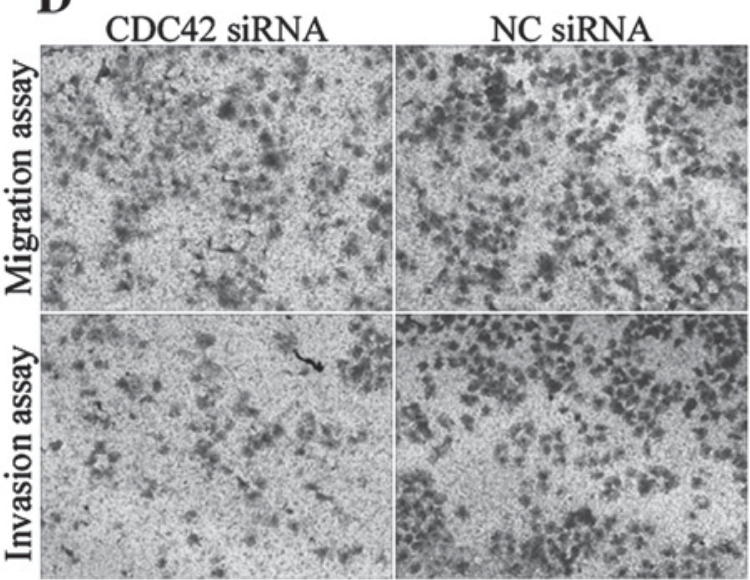

H522

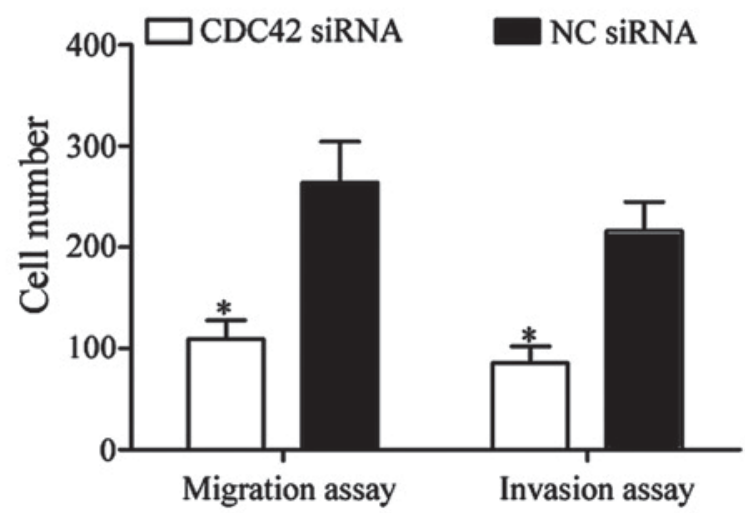

E

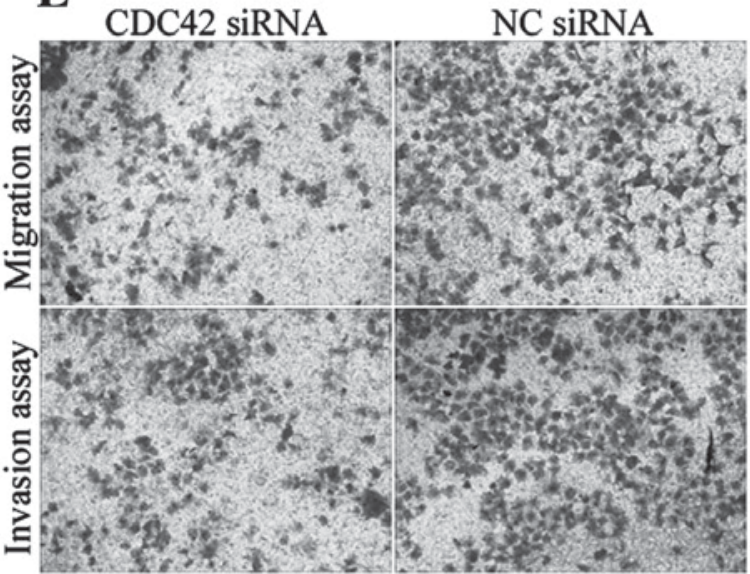

A549

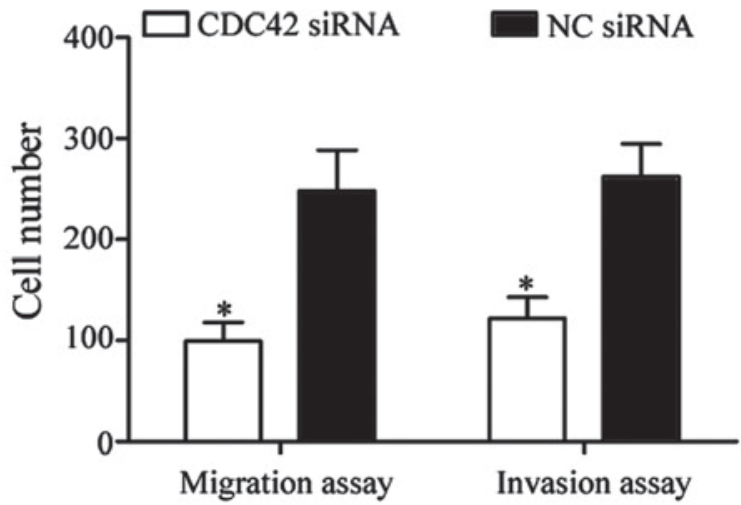

Figure 6. Effects of CDC42 in H522 and A549 cells. (A) Western blot analysis revealed that CDC42 was upregulated in H522 and A549 cells compared with BEAS-2B cells. "P<0.05 vs. BEAS-2B cells. GAPDH was used as a loading control. (B) CDC42 was downregulated in H522 and A549 cells transfected with CDC42 siRNA. GAPDH was used as a loading control. (C) CDC42 siRNA significantly inhibited H522 and A549 cell proliferation. "P<0.05 vs. NC siRNA. (D) CDC42 siRNA suppressed H522 cell migration and invasion. "P<0.05 vs. NC siRNA. (E) CDC42 siRNA suppressed A549 cell migration and invasion. "P<0.05 vs. NC siRNA. CDC42, cell division cycle 42; siRNA, small interfering RNA; NC, negative control. 
the functions of miR-29a were variable in tumors and exhibited tissue-specificity. These results also indicate that miR-29a may serve important roles in these types of cancer and may function as a potential therapeutic target gene.

In the present study, it was demonstrated that miR-29a inhibited NSCLC cell proliferation, migration and invasion. Since miR-29a is involved in NSCLC carcinogenesis and progression, the potential molecular mechanism contributing to miR-29a-induced inhibition of NSCLC proliferation, migration and invasion was investigated. In the present study, an important molecular link between miR-29a and CDC42 was determined in NSCLC. TargetScan and miRanda predicted that CDC42 was a direct target gene of miR-29a. Two putative miR-29a-binding sites were identified covering nucleotides 578-584 and 997-1004 of the CDC42 3'UTR. A dual-luciferase reporter assay demonstrated that miR-29a was directly targeted to the CDC42 3'UTR. RT-qPCR and western blot analysis demonstrated that miR-29a negatively regulated CDC42 expression at the post-transcriptional level. CDC42 was verified to be upregulated in NSCLC cell lines, in accordance with the downregulation of miR-29a. Knockdown of CDC42 also inhibited NSCLC cell proliferation, migration and invasion. These results suggest that miR-29a targets CDC42 to suppress NSCLC cell proliferation, migration and invasion. Identification of the target genes of miR-29a is important to understand its role in the initiation and progression of NSCLC, and also for exploring new targeted therapies for NSCLC.

CDC42, a member of the Rho family of GTPases, is located at $1 \mathrm{p} 36.1$ and encodes a $25-\mathrm{kDa}$ protein (38). It was initially identified in Saccharomyces cerevisiae as a cell-cycle mutant that contributed to the regulation of budding and mating projection (39). Previous studies have demonstrated that CDC42 is involved in cell proliferation, cell cycle progression, cytoskeletal remodeling, migration and invasion (40-43). Consistent with its important functions in these distinct physiological and pathological processes, abnormal expression of CDC42 has been identified in numerous diseases, particularly in human cancer. CDC42 has been identified to be upregulated in a number of human cancers, including lung cancer (44). Therefore, it may be advantageous to investigate novel targeted therapy against CDC42 in lung cancer. In the present study, it was identified that miR-29a negatively regulated CDC42 to inhibit NSCLC cell proliferation, migration and invasion. Therefore, miR-29a may be investigated as a targeted therapy for NSCLC.

CDC42 has been identified to be regulated by multiple miRNAs in numerous types of cancer. For example, in colorectal cancer, miR-137 decreases cell invasion and increases cell apoptosis by targeting CDC42 (45). In addition, miR-18a targets CDC42 to function as a tumor suppressor (46). Furthermore, miR-224 inhibits cell proliferation and invasion via blockade of CDC42 (47). In esophageal squamous cell carcinoma, miR-195 suppressed cell proliferation and invasion via targeting CDC42 (48). In hepatocellular carcinoma, miR-224 promotes cell proliferation, migration and invasion, and inhibited apoptosis by regulating CDC42 expression (49). In gastric cancer, CDC42 was demonstrated to be a direct gene of miR-137 (50). In NSCLC, miR-25 and miR-137 were demonstrated, by targeting CDC42, to be important regulators in initiation and progression and NSCLC $(51,52)$. To the best of our knowledge, the present study demonstrates for the first time that miR-29a serves a suppressive role in NSCLC proliferation and motility by directly targeting CDC42. miR-29a/ CDC42-based targeted therapy may be a novel treatment for NSCLC.

In conclusion, the results of the present study revealed that miR-29a was significantly downregulated in NSCLC and that decreased expression of miR-29a was associated with advanced TNM classification and metastasis. In addition, inhibition of miR-29a and knockdown of CDC42 decreased NSCLC cell proliferation, migration and invasion. Furthermore, miR-29a negatively regulated the expression of CDC42 at the post-transcriptional level. These results indicate that miR-29a targets CDC42 to inhibit NSCLC carcinogenesis and progression. Therefore, miR-29a may be investigated as a targeted therapy for NSCLC. Further study is required to address the potential of miR-29a as an NSCLC therapy.

\section{References}

1. Ilic N, Petricevic A, Arar D, Kotarac S, Banovic J, Ilic NF Tripkovic A and Grandic L: Skip mediastinal nodal metastases in the IIIa/N2 non-small cell lung cancer. J Thorac Oncol 2: 1018-1021, 2007.

2. Siegel R, Ma J, Zou Z and Jemal A: Cancer statistics, 2014. CA Cancer J Clin 64: 9-29, 2014.

3. Siegel RL, Miller KD and Jemal A: Cancer statistics, 2015. CA Cancer J Clin 65: 5-29, 2015.

4. Boffetta $\mathrm{P}$ and Nyberg F: Contribution of environmental factors to cancer risk. Br Med Bull 68: 71-94, 2003.

5. Didkowska J, Manczuk M, McNeill A, Powles J and Zatonski W: Lung cancer mortality at ages 35-54 in the European Union: Ecological study of evolving tobacco epidemics. BMJ 331: 189-191, 2005.

6. Ridge CA, McErlean AM and Ginsberg MS: Epidemiology of lung cancer. Semin Intervent Radiol 30: 93-98, 2013.

7. Paliogiannis P, Attene F, Cossu A, Budroni M, Cesaraccio R, Tanda F, Trignano M and Palmieri G: Lung cancer epidemiology in North Sardinia, Italy. Multidiscip Respir Med 8: 45, 2013.

8. Ni T, Mao G, Xue Q, Liu Y, Chen B, Cui X, Lv L, Jia L, Wang Y and Ji L: Upregulated expression of ILF2 in non-small cell lung cancer is associated with tumor cell proliferation and poor prognosis. J Mol Histol 46: 325-335, 2015.

9. Peters S, Adjei AA, Gridelli C, Reck M, Kerr K and Felip E; ESMO Guidelines Working Group: Metastatic non-small-cell lung cancer (NSCLC): ESMO clinical practice guidelines for diagnosis, treatment and follow-up. Ann Oncol 23 (Suppl 7): vii56-vii64, 2012.

10. Zhang T, Zhang DM, Zhao D, Hou XM, Liu XJ, Ling XL and Ma SC: The prognostic value of osteopontin expression in non-small cell lung cancer: A meta-analysis. J Mol Histol 45: 533-540, 2014.

11. Cai J, Fang L, Huang Y, Li R, Yuan J, Yang Y, Zhu X, Chen B, Wu J and Li M: miR-205 targets PTEN and PHLPP2 to augment AKT signaling and drive malignant phenotypes in non-small cell lung cancer. Cancer Res 73: 5402-5415, 2013

12. Pisters KM, Evans WK, Azzoli CG, Kris MG, Smith CA, Desch CE, Somerfield MR, Brouwers MC, Darling G, Ellis PM, et al: Cancer care ontario and American society of clinical oncology adjuvant chemotherapy and adjuvant radiation therapy for stages I-IIIA resectable non small-cell lung cancer guideline. J Clin Oncol 25: 5506-5518, 2007.

13. Verdecchia A, Francisci S, Brenner H, Gatta G, Micheli A, Mangone L and Kunkler I; EUROCARE-4 Working Group: Recent cancer survival in Europe: A 2000-02 period analysis of EUROCARE-4 data. Lancet Oncol 8: 784-796, 2007.

14. Sun W, Yuan X, Tian Y, Wu H, Xu H, Hu G and Wu K: Non-invasive approaches to monitor EGFR-TKI treatment in non-small-cell lung cancer. J Hematol Oncol 8: 95, 2015.

15. Liu R, Liu X, Zheng Y, Gu J, Xiong S, Jiang P, Jiang X, Huang E, Yang Y, Ge D and Chu Y: MicroRNA-7 sensitizes non-small cell lung cancer cells to paclitaxel. Oncol Lett 8: 2193-2200, 2014. 
16. Ma Y, Li X, Cheng S, Wei W and Li Y: MicroRNA-106a confers cisplatin resistance in non-small cell lung cancer A549 cells by targeting adenosine triphosphatase-binding cassette A1. Mol Med Rep 11: 625-632, 2015.

17. Zhong Z, Xia Y, Wang P, Liu B and Chen Y: Low expression of microRNA-30c promotes invasion by inducing epithelial mesenchymal transition in non-small cell lung cancer. Mol Med Rep 10: 2575-2579, 2014.

18. Shi Y, Liu C, Liu X, Tang DG and Wang J: The microRNA miR-34a inhibits non-small cell lung cancer (NSCLC) growth and the CD44hi stem-like NSCLC cells. PLoS One 9: e90022, 2014.

19. Li X, Abdel-Mageed AB, Mondal D and Kandil E: MicroRNA expression profiles in differentiated thyroid cancer, a review. Int J Clin Exp Med 6: 74-80, 2013.

20. Hwang HW and Mendell JT: MicroRNAs in cell proliferation, cell death, and tumorigenesis. Br J Cancer 96 (Suppl): R40-R44, 2007.

21. Tafsiri E, Darbouy M, Shadmehr MB, Zagryazhskaya A, Alizadeh $\mathrm{J}$ and Karimipoor $\mathrm{M}$ : Expression of miRNAs in non-small-cell lung carcinomas and their association with clinicopathological features. Tumour Biol 36: 1603-1612, 2015.

22. Braun J and Hüttelmaier S: Pathogenic mechanisms of deregulated microRNA expression in thyroid carcinomas of follicular origin. Thyroid Res 4 (Suppl 1): S1, 2011.

23. Zhang B, Pan X, Cobb GP and Anderson TA: microRNAs as oncogenes and tumor suppressors. Dev Biol 302: 1-12, 2007.

24. Esquela-Kerscher A and Slack FJ: Oncomirs-microRNAs with a role in cancer. Nat Rev Cancer 6: 259-269, 2006.

25. Livak KJ and Schmittgen TD: Analysis of relative gene expression data using real-time quantitative PCR and the 2(-Delta Delta C(T)) Method. Methods 25: 402-408, 2001.

26. Kriegel AJ, Liu Y, Fang Y, Ding X and Liang M: The miR-29 family: Genomics, cell biology, and relevance to renal and cardiovascular injury. Physiol Genomics 44: 237-244, 2012.

27. Wang Y, Zhang X, Li H, Yu J and Ren X: The role of miRNA-29 family in cancer. Eur J Cell Biol 92: 123-128, 2013.

28. Li J, Wan X, Qiang W, Li T, Huang W, Huang S, Wu D and Li Y: MiR-29a suppresses prostate cell proliferation and induces apoptosis via KDM5B protein regulation. Int J Clin Exp Med 8: $5329-5339,2015$.

29. Liu X, Cai J, Sun Y, Gong R, Sun D, Zhong X, Jiang S, He X, Bao E, Yang L and Li Y: MicroRNA-29a inhibits cell migration and invasion via targeting Roundabout homolog 1 in gastric cancer cells. Mol Med Rep 12: 3944-3950, 2015.

30. Liu C, Duan P, Li B, Huang C, Jing Y and Yan W: miR-29a activates Hes1 by targeting Nfia in esophageal carcinoma cell line TE-1. Oncol Lett 9: 96-102, 2015.

31. Wang F, Wang XS, Yang GH, Zhai PF, Xiao Z, Xia LY, Chen LR, Wang Y, Wang XZ, Bi LX, et al: miR-29a and miR-142-3p downregulation and diagnostic implication in human acute myeloid leukemia. Mol Biol Rep 39: 2713-2722, 2012

32. Zhu XC, Dong QZ, Zhang XF, Deng B, Jia HL, Ye QH, Qin LX and Wu XZ: microRNA-29a suppresses cell proliferation by targeting SPARC in hepatocellular carcinoma. Int J Mol Med 30: 1321-1326, 2012

33. Tang W, Zhu Y, Gao J, Fu J, Liu C, Liu Y, Song C, Zhu S, Leng Y, Wang G, et al: MicroRNA-29a promotes colorectal cancer metastasis by regulating matrix metalloproteinase 2 and E-cadherin via KLF4. Br J Cancer 110: 450-458, 2014.

34. Nishikawa R, Chiyomaru T, Enokida $H$, Inoguchi $S$, Ishihara $T$, Matsushita R, Goto Y, Fukumoto I, Nakagawa M and Seki N: Tumour-suppressive microRNA-29s directly regulate LOXL2 expression and inhibit cancer cell migration and invasion in renal cell carcinoma. FEBS Lett 589: 2136-2145, 2015.
35. Tréhoux S, Lahdaoui F, Delpu Y, Renaud F, Leteurtre E, Torrisani J, Jonckheere N and Van Seuningen I: Micro-RNAs miR-29a and miR-330-5p function as tumor suppressors by targeting the MUC1 mucin in pancreatic cancer cells. Biochim Biophys Acta 1853: 2392-2403, 2015.

36. Zhao Z, Wang L, Song W, Cui H, Chen G, Qiao F, Hu J, Zhou R and Fan H: Reduced miR-29a-3p expression is linked to the cell proliferation and cell migration in gastric cancer. World J Surg Oncol 13: 101, 2015.

37. Lu L, Xue X, Lan J, Gao Y, Xiong Z, Zhang H, Jiang W, Song W and Zhi Q: MicroRNA-29a upregulates MMP2 in oral squamous cell carcinoma to promote cancer invasion and anti-apoptosis. Biomed Pharmacother 68: 13-19, 2014.

38. Ma J, Xue Y, Liu W, Yue C, Bi F, Xu J, Zhang J, Li Y, Zhong C and Chen Y: Role of activated Rac1/Cdc42 in mediating endothelial cell proliferation and tumor angiogenesis in breast cancer. PLoS One 8: e66275, 2013.

39. Sun N, Ye L, Chang T, Li X and Li X: microRNA-195-Cdc42 axis acts as a prognostic factor of esophageal squamous cell carcinoma. Int J Clin Exp Pathol 7: 6871-6879, 2014.

40. Reymond N, Im JH, Garg R, Vega FM, Borda d'Agua B, Riou P, Cox S, Valderrama F, Muschel RJ and Ridley AJ: Cdc42 promotes transendothelial migration of cancer cells through $\beta 1$ integrin. J Cell Biol 199: 653-668, 2012.

41. Stengel KR and Zheng Y: Essential role of Cdc42 in Ras-induced transformation revealed by gene targeting. PLoS One 7: e37317, 2012.

42. Nobes CD and Hall A: Rho, rac and cdc42 GTPases regulate the assembly of multimolecular focal complexes associated with actin stress fibers, lamellipodia, and filopodia. Cell 81: 53-62, 1995.

43. Olson MF, Ashworth A and Hall A: An essential role for Rho, Rac and Cdc42 GTPases in cell cycle progression through G1. Science 269: 1270-1272, 1995.

44. Chen QY, Jiao DM, Yao QH, Yan J, Song J, Chen FY, Lu GH and Zhou JY: Expression analysis of Cde 42 in lung cancer and modulation of its expression by curcumin in lung cancer cell lines. Int J Oncol 40: 1561-1568, 2012.

45. Liu M, Lang N, Qiu M, Xu F, Li Q, Tang Q, Chen J, Chen X, Zhang S, Liu Z, et al: miR-137 targets Cdc 42 expression, induces cell cycle G1 arrest and inhibits invasion in colorectal cancer cells. Int J Cancer 128: 1269-1279, 2011.

46. Humphreys KJ, McKinnon RA and Michael MZ: miR-18a inhibits $\mathrm{CDC} 42$ and plays a tumour suppressor role in colorectal cancer cells. PLoS One 9: e112288, 2014.

47. Ke TW, Hsu HL, Wu YH, Chen WT, Cheng YW and Cheng CW: MicroRNA-224 suppresses colorectal cancer cell migration by targeting Cdc42. Dis Markers 2014: 617150, 2014.

48. Fu MG, Li S, Yu TT, Qian LJ, Cao RS, Zhu H, Xiao B, Jiao CH, Tang NN, Ma JJ, et al: Differential expression of miR-195 in esophageal squamous cell carcinoma and miR-195 expression inhibits tumor cell proliferation and invasion by targeting of Cdc42. FEBS Lett 587: 3471-3479, 2013.

49. Zhang Y, Takahashi S, Tasaka A, Yoshima T, Ochi H and Chayama K: Involvement of microRNA-224 in cell proliferation, migration, invasion, and anti-apoptosis in hepatocellular carcinoma. J Gastroenterol Hepatol 28: 565-575, 2013.

50. Chen Q, Chen X, Zhang M, Fan Q, Luo S and Cao X: miR-137 is frequently down-regulated in gastric cancer and is a negative regulator of Cdc42. Dig Dis Sci 56: 2009-2016, 2011.

51. Yang T, Chen T, Li Y, Gao L, Zhang S, Wang T and Chen M: Downregulation of miR-25 modulates non-small cell lung cancer cells by targeting CDC42. Tumour Biol 36: 1903-1911, 2015.

52. Zhu X, Li Y, Shen H, Li H, Long L, Hui L and Xu W: miR-137 inhibits the proliferation of lung cancer cells by targeting Cdc42 and Cdk6. FEBS Lett 587: 73-81, 2013. 